\title{
Biomedical Image Classification with Random Subwindows and Decision Trees
}

\section{Abstract}

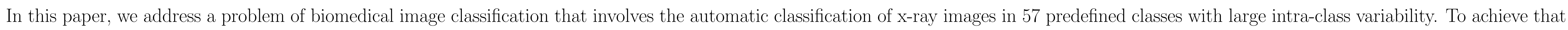

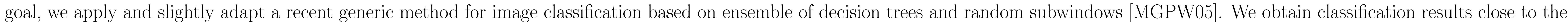
state of the art on a publicly available database of 10000 x-ray images. We also provide some clues to interpret the classification of each image in terms of subwindow relevance.

\section{Image Classification}

$\triangleright$ Goal:

Given a set of training images labelled into a finite number of classes, the goal of an automatic image classification method is to build a model that will be able to predict accurately the class of new, unseen images.

\section{Biomedical applications:}

Organize large-scale image databases into categories without limitation to a specific diagnostic study, setup clinical diagnosis tools, provide high-throughput cell phenotype screening,

$\triangleright$ Some solutions:

- Pre-processing "feature extraction" step, specific to the particular problem and application domain

- Features used as new input variables for traditional learning algorithms (nearest neighbor or neural network classifiers)

\section{Random Subwindows and Decision Trees}

\section{Concepts}

- Extraction of a large number of possibly overlapping, square subwindows of random sizes and at random posi-

tions

- Pixel-based description with scale normalization

- Tree-based machine learning ensemble methods

- Successfully applied to household objects, buildings, landscape themes, handwritten digits, faces,

Learning stage

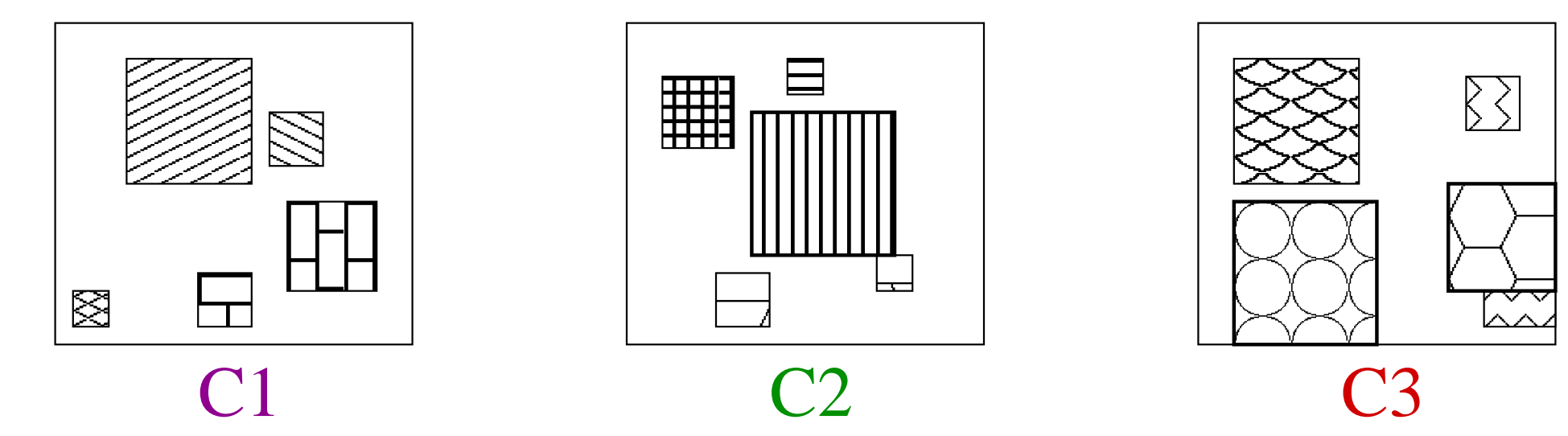

C1C1 C1 C1C1 $\mathrm{C} 2 \mathrm{C} 2 \mathrm{C} 2 \mathrm{C} 2 \mathrm{C} 2 \mathrm{C} 3 \mathrm{C} 3 \mathrm{C} 3 \mathrm{C} C \mathrm{C}$

- Class-balanced extraction of $\left(N_{w}\right)$ subwindows: from each training image of class $c$, we extract $N_{w} /\left(m * n b_{c}\right)$ subwindows where $m$ is the number of classes and $n b_{c}$ the number of training images of class $c$

- Subwindow resizing down to a fixed size $(16 \times 16$ pixels $)$

- Subwindow labeling as its parent image class

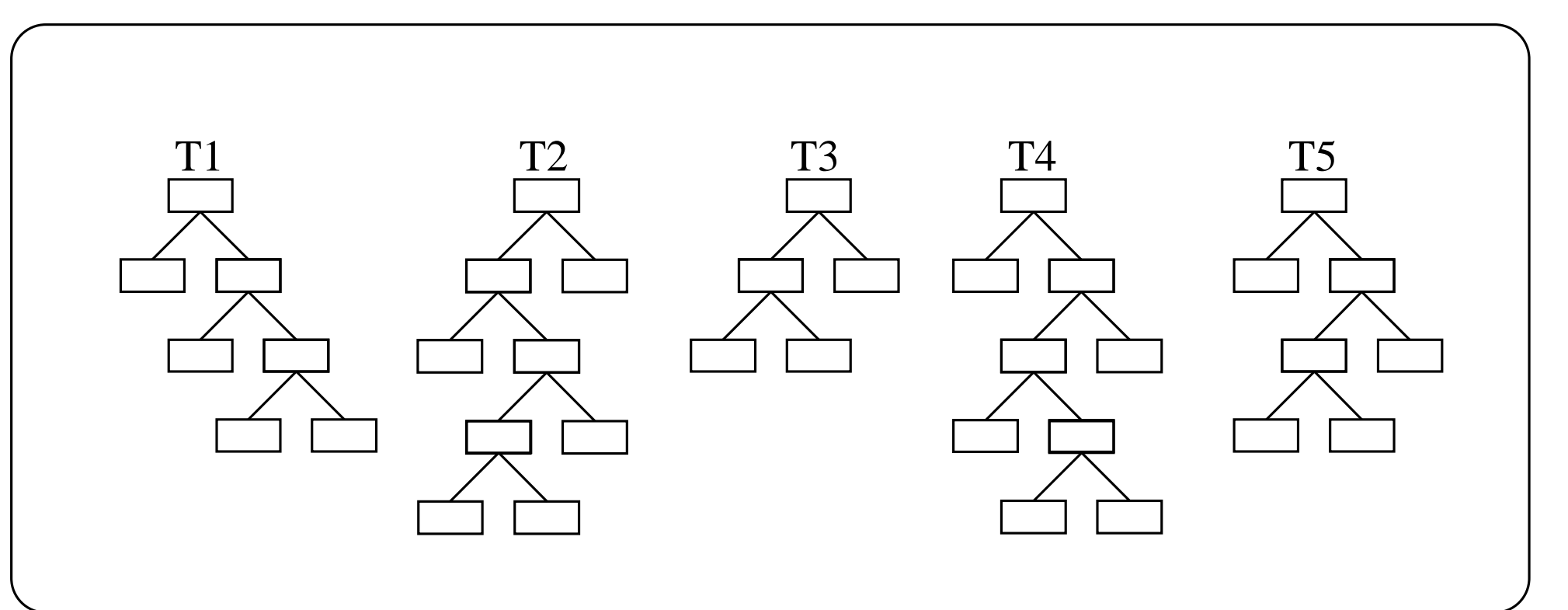

- Building a subwindow classification model using supervised methods

- Ensemble of $T$ decision tree methods: Tree Boosting, Extra-Trees [GEW06]
Random Subwindows and Decision Trees (continued)

Prediction stage
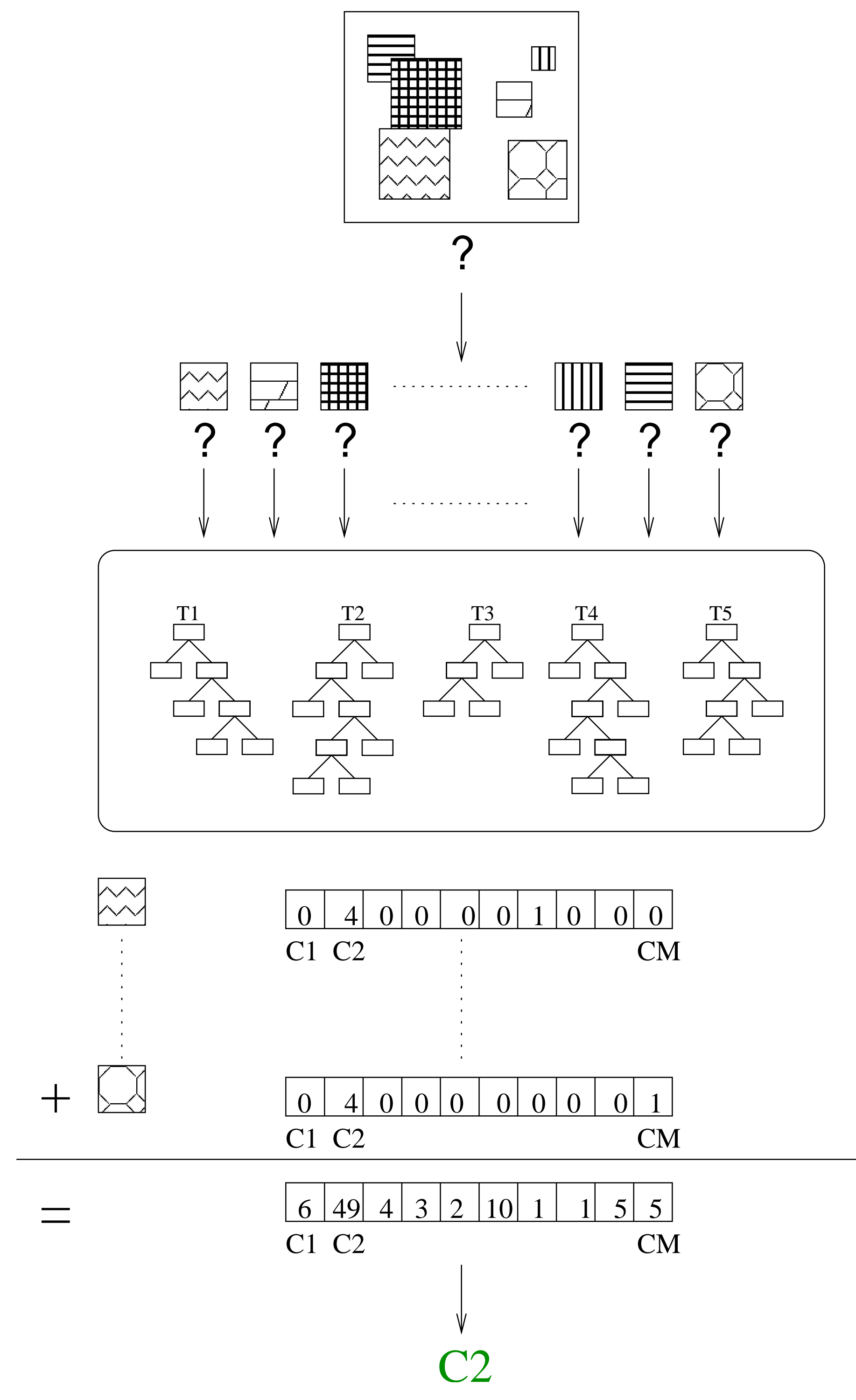

- Extraction of $N_{w, t e s t}$ subwindows in test image

- Propagation of each subwindow into each tree

- Aggregation of tree votes. We assign to the image the majority class among the classes assigned to its subwindows.

Dataset: IRMA challenge

Description

- 10000 x-ray images (courtesy of TM Lehmann, RWTH, Aachen, Germany, http://www.irma-project.org)

- 57 classes according to the IRMA code: different modalities, orientations, body parts, and biological systems Examples
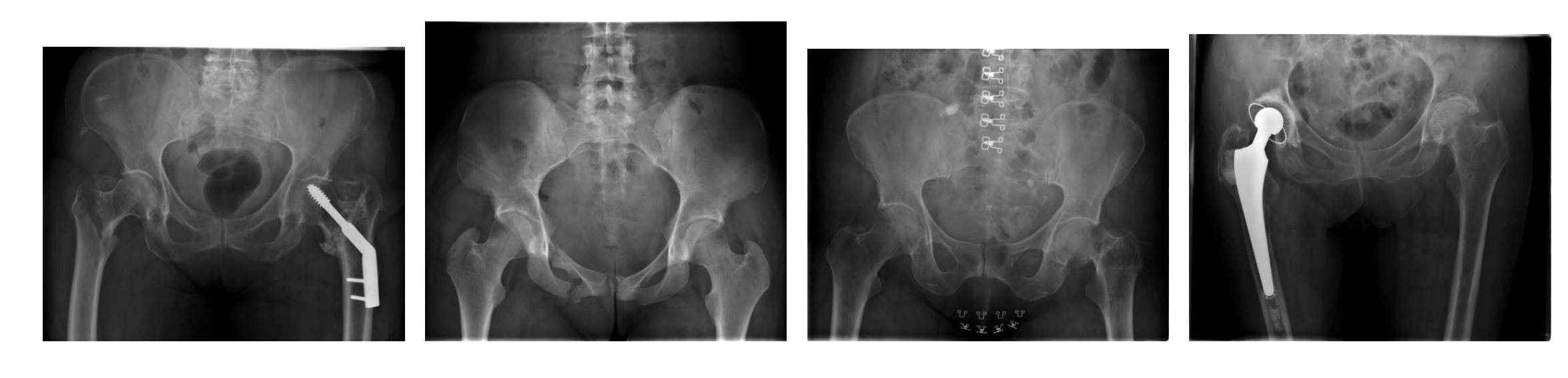

Images from the "coronal, pelvis, musculosceletal" class
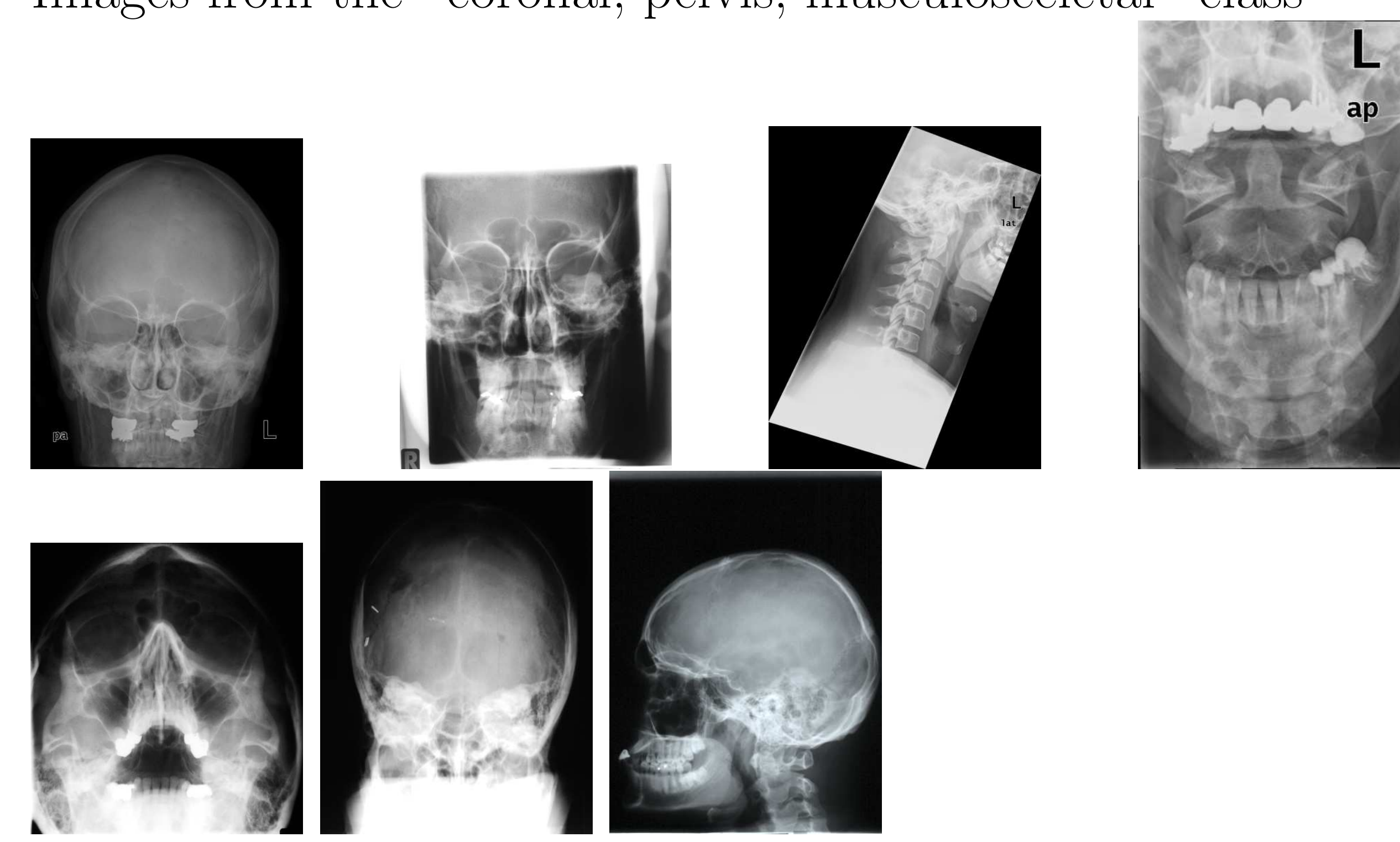

Images from 7 cranium/cervical spine classes

\section{Protocol and Results}

$\triangleright$ Protocol and parameters

- Training set: 9000 images, test set: 1000 images ([iCS05])

- $N_{w}=800000, T=25, N_{w, t e s t}=500$

$\triangleright$ Misclassification error rate

Method error rate

\begin{tabular}{l|l}
\hline $1-\mathrm{NN}+\mathrm{IDM}[\mathrm{KGN04}]$ & $12.6 \%$ \\
\hline
\end{tabular}

$\begin{array}{ll}1-\mathrm{NN}+\mathrm{CCF}+\mathrm{IDM}+\text { Tamura } & 13.3 \%\end{array}$

\begin{tabular}{ll} 
Discriminative patches [DKN05] $\quad 13.9 \%$ \\
\hline Res
\end{tabular}

Random Subwindows + Tree Boosting $14.0 \%$

\begin{tabular}{l|l} 
MI1 Confidence & $14.6 \%$ \\
\hline
\end{tabular}

Random Subwindows + Extra-Trees $\quad 14.7 \%$

\begin{tabular}{|l|l|}
\hline Gift $5 \mathrm{NN} 8 \mathrm{~g}$ & $20.6 \%$ \\
\hline
\end{tabular}

\begin{tabular}{ll} 
Nearest Neighbor, $32 \times 32$, Euclidian $\quad 36.8 \%$ \\
\hline
\end{tabular}

Texture directionality

$73.3 \%$

\section{Computational Efficiency}

- Training algorithm is on the order of $T N_{w} \log N_{w}$

- Prediction essentially proportional to $T N_{w, t e s t} \log N_{w}$

\section{Interpretability}

- Well classified subwindows could bring potentially useful information about that class

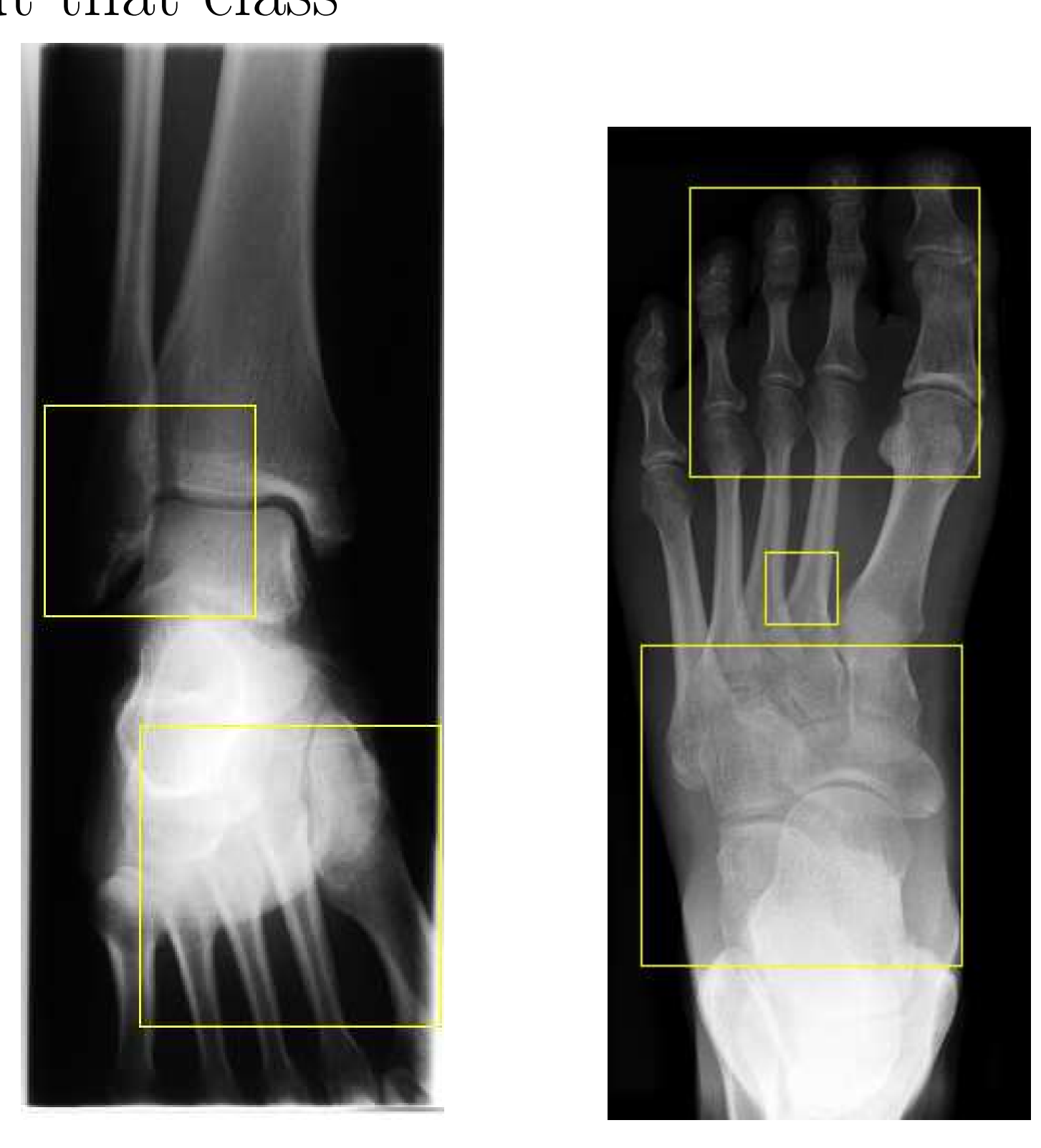

\section{Conclusion}

- We applied our generic method [MGPW05] on a specific biomedical task

- We obtained results competitive with state-of-the-art algorithms without tedious adaptation. It confirms the potential of the approach for a wide range of applications.

- The possibility to extract interpretable information from images has been highlighted

\section{References}

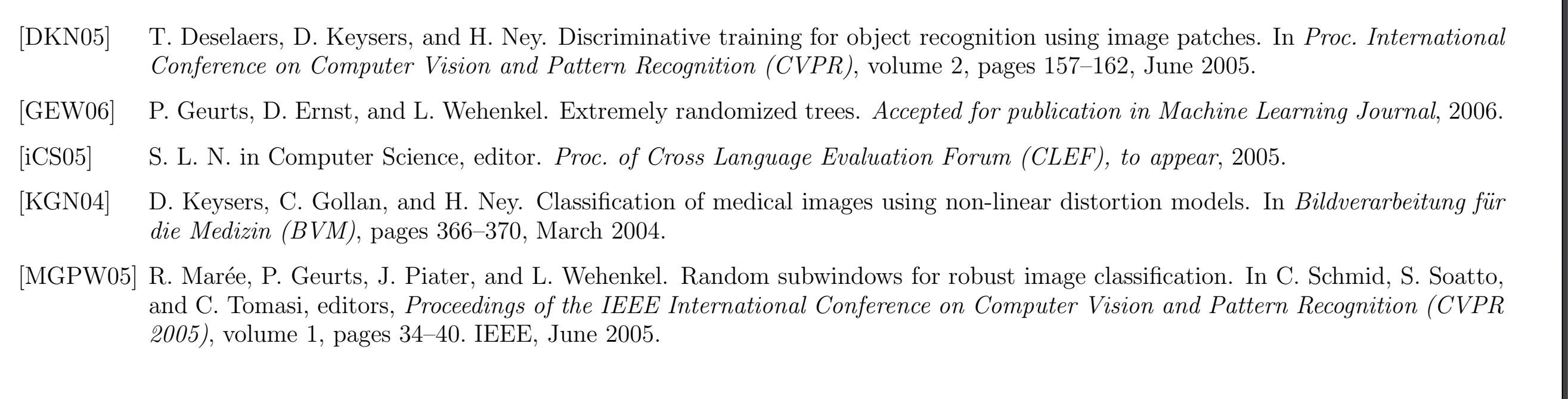

